\title{
MENSURAÇÃO DE PROBLEMAS LOCOMOTORES E DE LESÕES NO COXIM PLANTAR EM FRANGOS DE CORTE
}

\author{
MEASUREMENT OF LOCOMOTOR PROBLEMS AND PAD LESIONS IN BROILERS \\ Mendes, A.S. ${ }^{1 *}$, Paixão, S.J. ${ }^{2 A}$, Marostega, J. ${ }^{2 B}$, Restelatto, R. ${ }^{2 C}$, Oliveira, P.A.V. ${ }^{3}$ \\ e Possenti, J.C. ${ }^{1 A}$
}

\author{
${ }^{1}$ Universidade Tecnológica Federal do Paraná (UTFPR). Campus Dois Vizinhos-PR. Brasil. \\ *angelica@utfpr.edu.br; Ajpossenti@utfpr.edu.br \\ ${ }^{2}$ Zootecnia. UTFPR. Brasil. Asandropaixa014@hotmail.com; Bjulimarostega@hotmail.com; \\ crestelattor@yahoo.com.br \\ 3Embrapa Suínos e Aves. Brasil. paolive@cnpsa.embrapa.br
}

\section{PALAVRAS CHAVE ADICIONAIS}

Metodologias. Bem-estar animal. Densidade. Avaliadores.

\section{RESUMO}

Este estudo foi conduzido na UTFPR, Campus Dois Vizinhos-PR, dividido em dois experimentos com 600 pintainhos de corte machos, de mesma linhagem (Cobb) e origem. O objetivo deste estudo foi avaliar distintas metodologias para mensuração de lesões no coxim plantar e lesões locomotoras em frangos de corte, bem como o efeito da densidade de alojamento na incidência de lesões. Para o experimento de incidência de problemas locomotores, em delineamento inteiramente casualizado e em esquema fatorial $3 \times 3 \times 2$ (avaliadores $\mathrm{x}$ densidade $\mathrm{x}$ metodologias de problemas de locomoção). Para o experimento de incidência de lesões no coxim plantar, as aves foram distribuídas em delineamento inteiramente casualizado e em esquema fatorial $3 \times 3 \times 3$ (avaliadores $x$ densidade $x$ metodologias de lesões no coxim plantar), com quatro repetições cada. Para a avaliação de cada metodologia adotada, foram utilizados três avaliadores distintos e previamente treinados sobre a aplicação dos métodos e sem qualquer interação entre si. As médias obtidas foram comparadas pelo Testes de Tukey ao nível de $5 \%$ de probabilidade do erro. A repetibilidade do método foi analisada pelos escores mensurados pelos avaliadores. O coeficiente de correlação intraclasse (ICC) foi aplicado com nível de significância de $5 \%$. Densidades de alojamento mais elevadas afetaram a intensidade de proble-

Recibido: 8-10-10. Aceptado: 7-11-11.

\section{AdDitional KEYWORDS}

Methods. Animal welfare. Density. Evaluators.

mas locomotores e de lesões no coxim plantar das aves, aumentando com o desenvolvimento das mesmas. No geral, as metodologias de mensuração de problemas locomotores e de lesão no coxim plantar avaliadas neste estudo são passíveis de aplicação, porém não tão acessíveis de serem interpretadas.

\section{SUMMARY}

This study was conducted in UTFPR, Campus Dois Vizinhos-PR and divided into two experiments with 600 male broilers of the same lineage and origin. The objective of this study was to evaluate different methodologies for measurement of footpad and locomotors lesions in broilers and the effect of housing density on the incidence of these injuries. For the experiment the incidence of locomotor problems in a completely randomized design and factorial $3 \times 3 \times 2$ (density $\times$ evaluators $\times$ methods of locomotor problems). For the experiment of footpad dermatitis, the birds were distributed in a completely randomized design and factorial $3 x$ $3 \times 3$ (density $x$ evaluators $x$ methods of footpad lesions), with four replicates. For the evaluation of each methodology, we used three different evaluators who were previously trained on the application of methods and without any interaction between them. The averages were compared by 
Tukey tests at $5 \%$ probability of error. The repeatability of the method was analyzed from scores measurements taken by evaluators. The intraclass correlation coefficient (ICC) was applied with a significant level of $5 \%$. Density of housing affects the higher intensity of locomotor problems and foot pad dermatitis of the birds, increasing their development. In general, the methods were not available to be interpreted and applied.

\section{INTRODUÇÃO}

Um dos problemas mais graves na produção de frangos de corte é a alta incidência de distúrbios locomotores, particularmente aqueles que levam à mobilidade condicionada ou claudicação (European Commission, 2000). O desenvolvimento de muitos desses distúrbios é relacionado com a seleção genética e a gestão para um rápido crescimento, uma vez que problemas locomotores são raros em linhagens criadas em sistemas semi-intensivos, mas comuns nas aves comerciais, matrizes de frangos, patos e perus criados em confinamento (Kestin et al., 1992).

Lesões no coxim plantar são um tipo de dermatite causada por contato (Greene et al., 1985) afetando a região plantar das patas. As lesões podem ser desenvolvidas em menos de uma semana (Ekstrand et al., 1997). $\mathrm{Na}$ fase inicial, podem ser vistas descolorações na região plantar. Lesões mais profundas, como necroses da epiderme, podem ser vistas por análises histológicas. Em casos mais graves, as lesões podem evoluir para úlceras com reações inflamatórias (Greene et al., 1985).

A avicultura moderna, com grande número de aves por área e avanços nas áreas da genética, nutrição e manejo está suscetível a altos índices de problemas locomotores, em proporção maior que em algumas décadas passadas. Em alguns casos, a incidência de problemas locomotores pode ser elevada e associada com altas taxas de mortalidade (Goliomytis et al., 2003; Havenstein et al., 2003). Além disso, metodologias de avaliação da capacidade locomotora podem ser previstas utilizando métodos que avaliam a dor e o desconforto das aves (McGeown et al., 1999; Danbury et al., 2000).

Essas observações induzem a população a concluir que lotes comerciais de frangos de corte, frequentemente apresentem altos percentuais de aves com lesões locomotoras e a acreditar que as indústrias produzem animais com baixo nível de bem-estar. Porém, estão aumentando as evidências de que fatores ambientais podem ter uma influência considerável na incidência de problemas locomotores em frangos de corte e essas anormalidades podem ser mantidas a níveis baixos sob adequado manejo (Dawkins et al., 2004; Cummings et al., 2005).

Como preocupações sobre o bem-estar animal têm se tornando marketing para a conquista de consumidores pelas indústrias de alimentos, faz-se necessário o uso de métodos apropriados para medir a incidência de problemas locomotores em lotes comerciais de frangos de corte. A avaliação visual da capacidade locomotora (ou seja, o gait score) oferece a vantagem de permitir a avaliação não-invasiva de um grande número de aves em um curto espaço de tempo. Uma metodologia precisa e acessível para mensurar lesões locomotoras em aviários comerciais é necessária para que se obtenha uma linguagem única sobre o nível de bemestar das aves.

Além disso, os métodos devem permitir que os lotes de frangos sejam avaliados em um período de tempo razoável. A metodologia do gait score foi desenvolvida para avaliar as condições locomotoras de matrizes e foram adaptadas para a avaliação de frangos de corte (Kestin et al., 1992; Garner et al., 2002). Os métodos utilizados por esses autores definem seis categorias de anormalidade locomotoras em uma escala ordinal de severidade. As diferenças entre as categorias são sutis o suficiente para, talvez, tornar a metodologia mais difícil e mais lenta do que o necessário, para um ambiente de produção comercial. Outros autores usaram 


\section{MENSURAÇÃO DE LESÕES LOCOMOTORAS EM FRANGOS DE CORTE}

menos categorias de pontuação para a capacidade locomotora, mas falham por não avaliar profundamente as lesões e se tornarem métodos não confiáveis de auditoria de bem-estar animal (Dawkins et al., 2004).

Desta forma, este estudo objetivou avaliar distintas metodologias para mensuração de lesões no coxim plantar e lesões locomotoras em frangos de corte, bem como o efeito da densidade de alojamento na incidência destas lesões.

\section{MATERIALE MÉTODOS}

Dois experimentos foram conduzidos no setor de Avicultura da Universidade Tec- nológica Federal do Paraná, Campus Dois Vizinhos. Foram utilizados 600 pintainhos de corte machos, linhagem comercial Cobb ${ }^{\circledR}$ e provenientes de mesmo lote de matrizes, fornecidos por incubatório local.

\section{AVALIAÇÃODE PROBLEMASLOCOMOTORES}

As aves foram distribuídas em delineamento inteiramente casualizado, em fatorial 3 × 3 × 2 (densidade de alojamento $\times$ avaliadores $\mathrm{x}$ metodologias de problemas locomotores). Foram testadas três densidades de alojamento $\left(12,15\right.$ e 18 aves $\left./ \mathrm{m}^{2}\right)$ e duas metodologias para problemas locomotores, com quatro repetições cada. A descrição das metodologias pode ser observada na tabela $\mathbf{I}$.

Tabela I. Descrição das metodologias adotas para avaliar problemas locomotores e lesões no coxim plantar das aves. (Description of the methodologies used to assess footpad and locomotor lesions of birds).

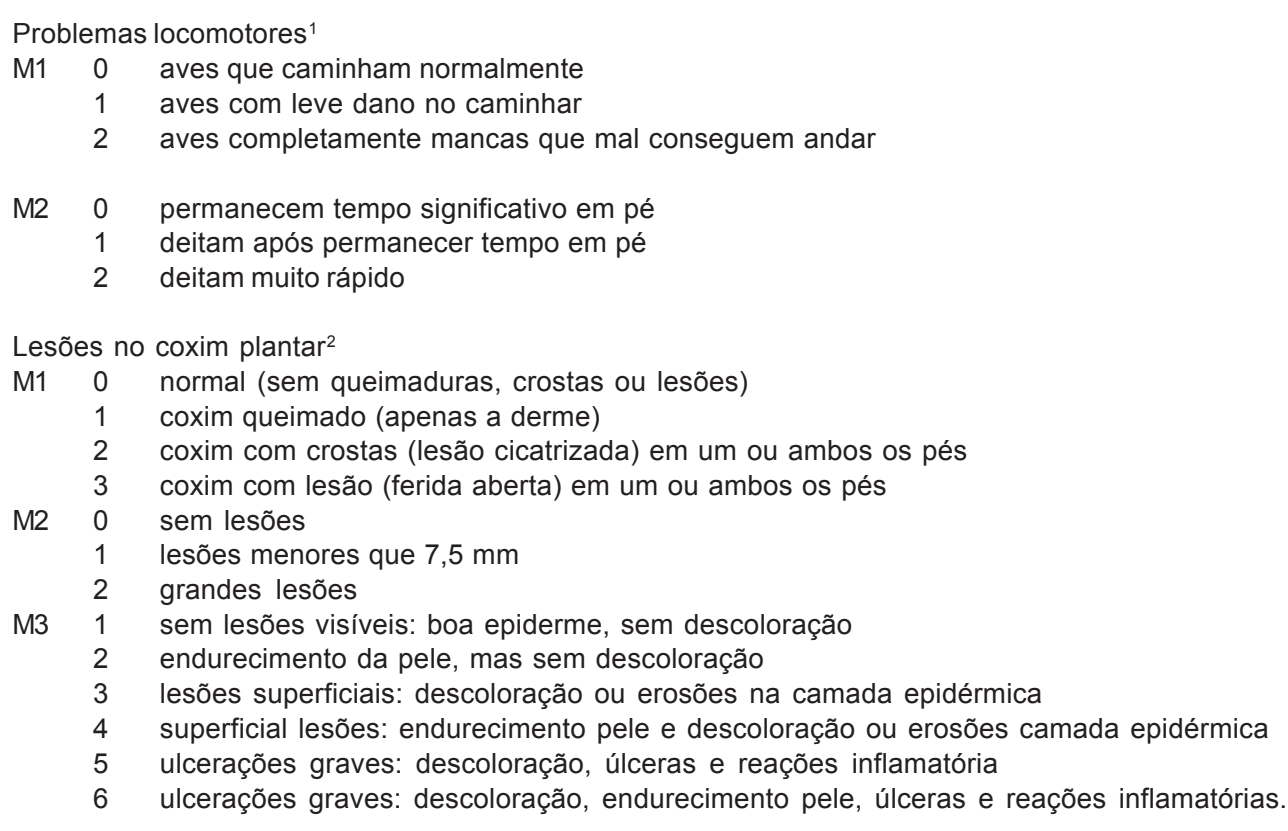

Metodologias e escores: ${ }^{1} \mathrm{M} 1=$ Kestin et al. (1992); M2= Berg e Sanotra (2003), as aves são colocadas individualmente em um recipiente com água (em altura que cubra os pés) e realiza-se a contagem do tempo em que elas levam para deitar; ${ }^{2} \mathrm{M} 1=\mathrm{McW}$ ard e Taylor (2000); M2= Bilgili et al. (1999); M3=Ekstrand (1994)

Archivos de zootecnia vol. 61, núm. 234, p. 219. 


\section{AVALIAÇÃO DE LESÕES NO COXIM PLANTAR}

As aves foram distribuídas em delineamento inteiramente casualizado, em fatorial $3 \times 3 \times 3$ (densidade de alojamento $x$ avaliadores $\mathrm{x}$ metodologias de lesões no coxim plantar). Foram testadas três densidades de alojamento (12, 15 e 18 aves $\left./ \mathrm{m}^{2}\right)$ e três metodologias para lesões no coxim plantar, com quatro repetições cada. A descrição das metodologias pode ser observada na tabela I.

Para ambos os experimentos as aves foram alocadas em 12 boxes $\left(2 \mathrm{~m}^{2}\right.$ cada $)$, de acordo com a densidade de cada nível deste fator, totalizando 300 aves em avaliação, conforme metodologia proposta por Neto e Campos (2002). Para a representação real do ambiente de alojamento, outras 300 aves foram alojadas nas áreas externas aos boxes.

As aves foram criadas durante 42 dias com água e alimentação à vontade. O fornecimento da ração foi realizado diariamente em três horários: 7, 13 e 18 h, juntamente com a limpeza dos bebedouros e com o manejo de cama sendo realizado uma vez ao dia.

Os 12 boxes foram equipados durante as fases iniciais com campânulas a gás, para aquecimento dos pintos e comedouros e bebedouros do tipo tubular infantil. O controle do aquecimento, bem como o manejo das cortinas, foi realizado de acordo com a necessidade das aves, com a aferição das condições ambientais realizada com o auxílio de termômetro digital alocado na altura das aves. Gradativamente, os equipamentos para a fase inicial foram substituídos por comedouros tubulares e bebedouros pendulares e, o aquecimento deixou de ser utilizado. O programa de luz adotado compreendeu 19 horas de iluminação por dia, em todas as fases de criação.

Iniciaram-se as avaliações das lesões semanalmente a partir dos 15 dias de idade das aves, todas no mesmo dia, totalizandose ao final cinco avaliações das lesões (15, $22,29,36$ e 42 dias). Cinco aves por box foram assinaladas no dorso com caneta pin- cel, garantindo que as mesmas aves fossem repetidamente avaliadas, até o término dos experimentos. Para a avaliação de cada metodologia adotada foram utilizados três avaliadores distintos e previamente treinados sobre a aplicação dos métodos, realizando-as no mesmo período e nas mesmas aves e, sem qualquer interação entre si. O treinamento foi idêntico entre os avaliadores, constando de 2 horas teóricas e de 6 horas práticas, efetuado no lote anterior ao de estudo.

Os dados obtidos foram analisados pela ANOVA (SAS, 2003) e as médias comparadas pelo teste de Tukey a $5 \%$ de probabilidade. Os dados foram transformados segundo $\log (\mathrm{x}+1)$, para reduzir a variação entre os escores das lesões. A análise estatística da confiabilidade interexaminadores e da repetibilidade do método foram averiguadas pelos resultados do índice de correlação intra-classe (ICC), considerando um nível de significância de p $<0,05$.

Conforme metodologia proposta por Iunes et al. (2005), um valor de ICC igual a 1 indica que os escores são idênticos nas comparações interexaminadores ou na repetibilidade do método. Valores de ICC abaixo de 0,7 são considerados não aceitáveis, entre 0,71 a 0,79 , aceitáveis, entre 0,8 a 0,89 , como muito bons e acima de 0,9 , excelentes. Também são apresentados os valores correspondentes a duas vezes o erro padrão dos escores mensurados na repetibilidade do método, ou seja, na comparação dos dados obtidos em registros distintos.

\section{RESULTADOSEDISCUSSÃO}

\section{EXPERIMENTO1}

\section{Avaliação de problemas locomotores}

De acordo com os resultados obtidos na análise de variância para as variáveis idade das aves e gait score não foi observado interação $(p<0,05)$ entre os fatores avaliados (densidade $\mathrm{x}$ avaliador $\mathrm{x}$ metodologia; 


\section{MENSURAÇÃO DE LESÕES LOCOMOTORAS EM FRANGOS DE CORTE}

densidade $\mathrm{x}$ avaliador; densidade $\mathrm{x}$ metodologia e avaliador x metodologia). Porém, quando se avaliou os fatores separadamente houve diferença $(\mathrm{p}<0,05)$ para o fator avaliador dentro das idades de 15,36 e 42 dias e do fator densidade de alojamento dentro das idades de 15 e 42 dias. Quanto ao fator metodologia dos problemas locomotores adotado, a diferença estatística foi constatada para todas as idades das aves (tabela II).

Para a densidade de alojamento, houve efeito $(p<0,05)$ na incidência de lesões no coxim plantar aos 15 e 42 dias de idade das aves, para todas as metodologias. Aos 15 dias houve maiores escores para a densidade de 12 aves $/ \mathrm{m}^{2}$, seguida pela de 18 aves $/ \mathrm{m}^{2}$ sem diferença entre si. Contudo esses resultados se modificaram com o crescimento das aves. Esse comportamento de incidência de lesões quando as aves são muito jovens, pode ser explicado pela fragilidade da derme dos pintainhos, que adquirem maior resistência com a idade e, com isso, diminuíram a incidência de lesões na idade intermediária do lote. Quando o lote atingiu a idade adulta, aos 42 dias, observou-se os maiores escores de lesões para a densidade de 18 aves $/ \mathrm{m}^{2}$. Esses resultados indicam que a densidade afeta a incidência de lesões no coxim plantar das aves. De acordo com Greene et al. (1985), as lesões podem se desenvolver em menos de uma semana e depois evoluir para úlceras principalmente nas aves adultas.

Trabalho realizado por Cravener et al. (1992), revela que acréscimos no número de aves por área ocasiona efeitos negativos no desempenho de frangos de corte, como aumento de lesões locomotoras, da umidade da cama, e de lesões no coxim plantar, influenciando diretamente na qualidade do produto final.

$\mathrm{O}$ efeito significativo constatado do

Tabela II. Gait score das lesões no coxim plantar, de acordo com os avaliadores, densidade de alojamento $\left(D 1=12, D 2=15\right.$ e $D 3=18$ aves $\left./ \mathrm{m}^{2}\right)$ e metodologia adotada. (Gait score for footpad lesions according to evaluators, density (D1= 12, D2 $=15$ e D3= 18 birds $/ \mathrm{m}^{2}$ ) and methodology).

\begin{tabular}{|c|c|c|c|c|c|c|}
\hline \multirow[b]{2}{*}{ Avaliador } & \multicolumn{5}{|c|}{ Idade das aves (dias) } & \multirow[b]{2}{*}{ Média } \\
\hline & 15 & 22 & 29 & 36 & 42 & \\
\hline 1 & $1,31^{\mathrm{a} 1}$ & $1,49^{a}$ & $1,59^{a}$ & $1,88^{a}$ & $2,6^{\mathrm{a}}$ & 1,77 \\
\hline 2 & $1,1^{b}$ & $1,41^{\mathrm{a}}$ & $1,59^{a}$ & $1,74^{\mathrm{ab}}$ & $2,53^{a}$ & 1,67 \\
\hline 3 & $0,85^{c}$ & $1,25^{\mathrm{a}}$ & $1,55^{\mathrm{a}}$ & $1,61^{b}$ & $2,16^{b}$ & 1,48 \\
\hline Média & 1,09 & 1,38 & 1,58 & 1,74 & 2,43 & \\
\hline Densidade (aves $\left./ \mathrm{m}^{2}\right)$ & 15 & 22 & 29 & 36 & 42 & \\
\hline 12 & $1,16^{\mathrm{a}}$ & $1,35^{\mathrm{a}}$ & $1,58^{a}$ & $1,83^{a}$ & $2,28^{b}$ & 1,64 \\
\hline 15 & $0,98^{b}$ & $1,35^{\mathrm{a}}$ & $1,52^{\mathrm{a}}$ & $1,68^{a}$ & $2,34^{b}$ & 1,57 \\
\hline 18 & $1,11^{\mathrm{ab}}$ & $1,46^{a}$ & $1,64^{a}$ & $1,71^{a}$ & $2,67^{a}$ & 1,72 \\
\hline Média & 1,08 & 1,39 & 1,58 & 1,74 & 2,43 & \\
\hline Metodologia & 15 & 22 & 29 & 36 & 42 & \\
\hline 1 & $0,87^{a}$ & $1,28^{a}$ & $1,54^{a}$ & $1,69^{a}$ & $2,37^{a}$ & 1,55 \\
\hline 2 & $0,68^{b}$ & $0,81^{b}$ & $1,01^{b}$ & $1,15^{b}$ & $1,6^{b}$ & 1,05 \\
\hline 3 & $1,88^{c}$ & $2,28^{c}$ & $2,35^{c}$ & $2,97^{c}$ & $2,58^{c}$ & 2,41 \\
\hline Média & 1,14 & 1,46 & 1,63 & 1,94 & 2,18 & \\
\hline CV (\%) & 19,4 & 27,57 & 15,07 & 13,25 & 8,67 & \\
\hline
\end{tabular}

${ }^{1}$ Médias seguidas por letras minúsculas na mesma coluna, não diferem significativamente ao nível de $5 \%$ de probabilidade pelo teste de Tukey. 
MENDES, PAIXÃO, MAROSTEGA, RESTELATTO, OLIVEIRAE POSSENTI

avaliador nas avaliações conduzidas aos 15,36 e 42 dias, para todas as metodologias, indica que as mesmas não são tão acessíveis de serem interpretadas e/ou um treinamento mais aprofundado necessita ser ministrado, pois os avaliadores obtiveram distintas interpretações do nível de bem-estar das aves. Além disso, houve divergências na aplicação do método no último período de avaliação, sendo este considerado o mais importante para aferição das lesões locomotoras, pois a ave está pronta para o abate e por ser mais pesada sofre mais com as lesões. Essas metodologias devem ser revistas, pois conforme Haslam e Kestin (2004), os métodos de avaliação devem ser simples, objetivos e que realmente representem o bem-estar do animal.

Em relação às metodologias aplicadas, verificou-se diferenças $(p<0,05)$ entre os resultados das avaliações para todas as fases de criação, o que leva a distintos diagnósticos. Esses resultados corroboram com Webster et al. (2008), em avaliação de diferentes metodologias, onde concluíram que os métodos com menores níveis de escores são mais precisos e com possível avaliação semelhante entre distintos avaliadores.

A análise dos valores de ICC (índice de correlação intra-classe) para a confiabilidade interexaminadores mostrou que os escores obtidos pelas distintas metodologias apresentaram níveis não aceitáveis de confiabilidade, para pelo menos um avaliador (tabela III).

Quanto ao comportamento da incidência de lesões no coxim plantar (figura 1), observou-se que à medida que as aves se desenvolveram aumentou-se a incidência das lesões no coxim plantar, para todas as metodologias. Pode-se constatar, também que, a mais alta densidade provocou maior incidência de lesões. Esse resultado está de acordo com Dawkins et al. (2004) os quais relataram que lesões no coxim plantar são mais predominantes em aves.

Dozier et al. (2005) relataram que o aumento de lesões devido a altas densidades e o crescimento das aves, é provavelmente um reflexo da baixa qualidade da cama. Já Sugeta et al. (2002), relataram que prejuízos no desempenho dos frangos de corte podem decorrer de diversos fatores, inclusive de programas de restrição alimentar e de ganhos compensatórios. Concordando com o exposto, Cordeiro et al. (2009) relataram que independente da metodologia empregada, o gait score aumentou a partir dos 35 dias de idade das aves.

Tabela III. Valores de ICC, duas vezes o erro padrão e nível de confiabilidade para os escores obtidos interexaminadores para as três metodologias. A média representa os escores obtidos a partir dos três avaliadores. (ICC values, twice standard error and level of reliability for the scores obtained (inter-examiner) for the three methodologies. The average represents the scores obtained from the three evaluators).

\begin{tabular}{cccccc}
\hline Avaliador & Metodologia & Média & ICC & 2 x erro-padrão & Nível \\
\hline 1 & 1 & 1,47 & 1,000 & 0,152 & Excelente \\
1 & 2 & 0,98 & 0,173 & 0,162 & Não aceitável \\
1 & 3 & 2,28 & 0,457 & 0,172 & Não aceitável \\
2 & 1 & 1,45 & 0,173 & 0,153 & Não aceitável \\
2 & 2 & 1,21 & 1,000 & 0,161 & Excelente \\
2 & 3 & 2,71 & 0,797 & 0,253 & Aceitável \\
3 & 1 & 1,81 & 0,457 & 0,157 & Não aceitável \\
3 & 2 & 1,07 & 0,797 & 0,188 & Aceitável \\
3 & 3 & 2,71 & 1,000 & 0,21 & Excelente \\
\hline
\end{tabular}

Archivos de zootecnia vol. 61, núm. 234, p. 222. 


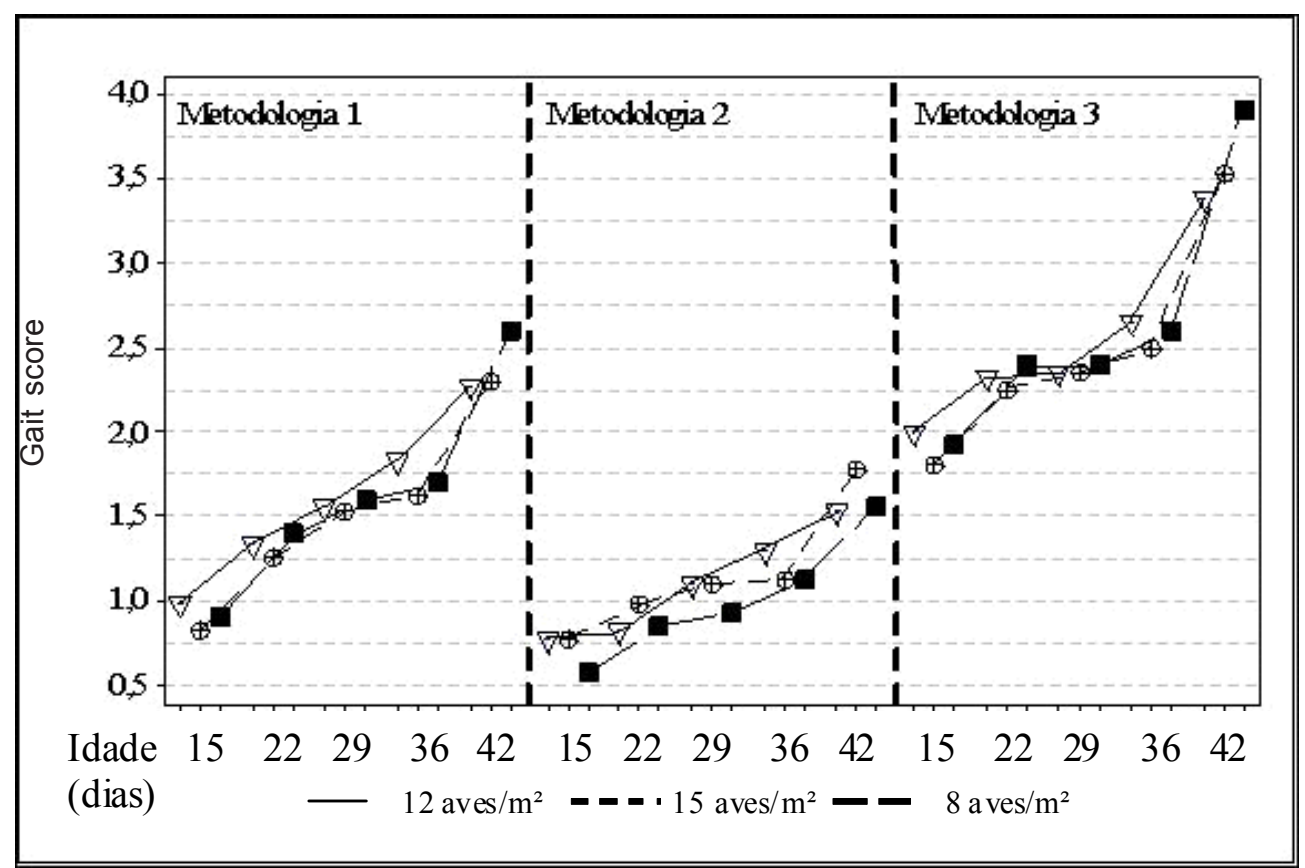

Figura 1. Escores médios obtidos pelos avaliadores para lesões no coxim plantar para as metodologias (M1, M2 e M3) e para cada densidade e idade das aves. (Mean scores obtained by the evaluators for footpad lesions for methodologies (M1, M2 and M3) and for each density and age of the birds).

\section{EXPERIMENTO2}

\section{Avaliação de lesões no coxim plantar}

Quanto à incidência de problemas locomotores nas aves observou-se diferenças $(\mathrm{p}<0,05)$ para a interação entre os fatores avaliador $x$ metodologia (tabela IV) apenas para a idade de 36 dias. $\mathrm{O}$ mesmo efeito $(\mathrm{p}<0,05)$ não foi observado nas demais idades avaliadas $(15,22,29$ e 42 dias $)$ quanto à interação entre os referidos fatores. Para as demais interações (densidade $\mathrm{x}$ avaliador $\mathrm{x}$ metodologia; densidade $\mathrm{x}$ avaliador e avaliador x metodologia), não constatou-se diferenças $(p<0,05)$ para as lesões no coxim plantar (tabela V).

As diferenças significativas entre os fatores avaliador $\mathrm{x}$ metodologia foi quando realizou-se a análise dos problemas locomotores aos 36 dias de idade das aves (tabela IV).
Tabela IV. Gait score de problemas locomotores de acordo com a metodologia empregada e o avaliador para a idade de 36 dias. (Gait score for locomotor lesions according to the methodology and the evaluator at age of 36 days).

\begin{tabular}{cccc}
\hline Avaliador & \multicolumn{2}{c}{ Metodologia } & Média \\
& 1 & 2 & \\
\hline 1 & $0,48^{\mathrm{Ab}}$ & $0,22^{\mathrm{Ba}}$ & 0,35 \\
2 & $0,93^{\mathrm{Aa}}$ & $0,11^{\mathrm{Ba}}$ & 0,52 \\
3 & $0,87^{\mathrm{Aa}}$ & $0,07^{\mathrm{Ba}}$ & 0,47 \\
Média & 0,76 & 0,13 & \\
\hline
\end{tabular}

${ }^{1}$ Médias seguidas por letras minúsculas na mesma coluna e maiúsculas na mesma linha, não diferem significativamente ao nível de $5 \%$ de probabilidade pelo teste de Tukey. 
Para a densidade de alojamento verificouse problemas locomotores em duas densidades aplicadas ( 15 e 18 aves $/ \mathrm{m}^{2}$ ) nas idades de avaliação de 15 e 42 dias. Contudo, analisando-se as aves aos 22, 29 e 36 dias de idade, as densidades utilizadas não diferiram $(p<0,05)$ entre si na avaliação dos problemas locomotores (tabela V).

Em geral, observou-se que a idade final de 42 dias apresentou os maiores escores de problemas locomotores para a densidade de 18 aves $/ \mathrm{m}^{2}$, independente da metodologia empregada. Esse resultado indica que a densidade afeta o desempenho locomotor das aves, principalmente as adultas, corroborando com Kestin et al. (1994), no qual relataram que aves com quatro semanas de idade apresentaram baixos índices de problemas locomotores em comparação com aves acima desta idade. Com isso, percebese que esses problemas desenvolvem-se no momento em que o lote atinge maior idade.

Estudos de Cravener et al. (1992) revelaram que o acréscimo na densidade de alojamento ocasiona efeitos negativos no desempenho de frangos como aumento da produção de amônia, de lesões locomotoras, da umidade da cama, do estresse calórico e dificuldade no empenamento. Sorensen et al. (2000) igualmente relataram maior umidade da cama e de lesões locomotoras em frangos, em tratamentos com maior número de aves por área. De acordo com Roll et al. (2010), outros fatores podem ocasionar efeitos negativos no desempenho e no bem-estar de frangos de corte como o estresse ou a restrição de uma preferência da espécie.

Outros estudos (Estevez et al., 1997) sobre padrões de atividade em frangos de corte têm indicado que grande parte dessa atividade locomotora diminuiu com a idade, enquanto que o tempo dispensado para descanso aumentou. Entretanto, diversos fatores, incluindo maior densidade de alojamento (Lewis e Hurnik, 1990; Estevez

Tabela $\boldsymbol{V}$. Gait score dos problemas locomotores, de acordo com os avaliadores, densidade de alojamento $\left(D 1=12, D 2=15\right.$ e D3 $=18$ aves $\left./ \mathrm{m}^{2}\right)$ e metodologia adotada. (Gait score for locomotor lesions according to the evaluators, density (D1 = 12, D2 $=15$ e D3= $\left.18 \mathrm{birds} / \mathrm{m}^{2}\right)$ and methodology).

\begin{tabular}{ccccccc}
\hline & \multicolumn{5}{c}{ Idade das aves (dias) } & \\
Avaliador & 15 & 22 & 29 & 36 & 42 & Média \\
\hline 1 & $0,47^{\mathrm{a} 1}$ & $0,31^{\mathrm{a}}$ & $0,43^{\mathrm{a}}$ & $0,46^{\mathrm{a}}$ & $0,52^{\mathrm{a}}$ & 0,44 \\
2 & $0,36^{\mathrm{a}}$ & $0,30^{\mathrm{a}}$ & $0,35^{\mathrm{a}}$ & $0,41^{\mathrm{a}}$ & $0,47^{\mathrm{a}}$ & 0,38 \\
3 & $0,36^{\mathrm{a}}$ & $0,26^{\mathrm{a}}$ & $0,35^{\mathrm{a}}$ & $0,25^{\mathrm{b}}$ & $0,41^{\mathrm{a}}$ & 0,33 \\
Média & 0,40 & 0,29 & 0,38 & 0,37 & 0,47 & \\
Densidade (aves $\left./ \mathrm{m}^{2}\right)$ & 15 & 22 & 29 & 36 & 42 & \\
12 & $0,24^{\mathrm{a}}$ & $0,31^{\mathrm{a}}$ & $0,43^{\mathrm{a}}$ & $0,31^{\mathrm{a}}$ & $0,38^{\mathrm{a}}$ & 0,33 \\
15 & $0,47^{\mathrm{b}}$ & $0,31^{\mathrm{a}}$ & $0,37^{\mathrm{a}}$ & $0,37^{\mathrm{a}}$ & $0,46^{\mathrm{ab}}$ & 0,40 \\
18 & $0,49^{\mathrm{b}}$ & $0,26^{\mathrm{a}}$ & $0,34^{\mathrm{a}}$ & $0,44^{\mathrm{a}}$ & $0,55^{\mathrm{b}}$ & 0,42 \\
Média & 0,40 & 0,29 & 0,38 & 0,37 & 0,46 & \\
Metodologia & 15 & 22 & 29 & 36 & 42 & \\
1 & $0,41^{\mathrm{a}}$ & $0,54^{\mathrm{a}}$ & $0,74^{\mathrm{a}}$ & $0,75^{\mathrm{a}}$ & $0,86^{\mathrm{a}}$ & 0,66 \\
2 & $0,39^{\mathrm{a}}$ & $0,08^{\mathrm{b}}$ & $0,09^{\mathrm{b}}$ & $0,08^{\mathrm{b}}$ & $0,15^{\mathrm{b}}$ & 0,16 \\
Média & 0,40 & 0,31 & 0,42 & 0,51 & 0,41 & \\
CV (\%) & 64,1 & 59,9 & 61,2 & 47,3 & 33,8 & \\
\hline
\end{tabular}

${ }^{1}$ Médias seguias por letras minúscula na mesma coluna, não diferem significativamente ao nível de $5 \%$ de probabilidade pelo teste de Tukey.

Archivos de zootecnia vol. 61, núm. 234, p. 224. 


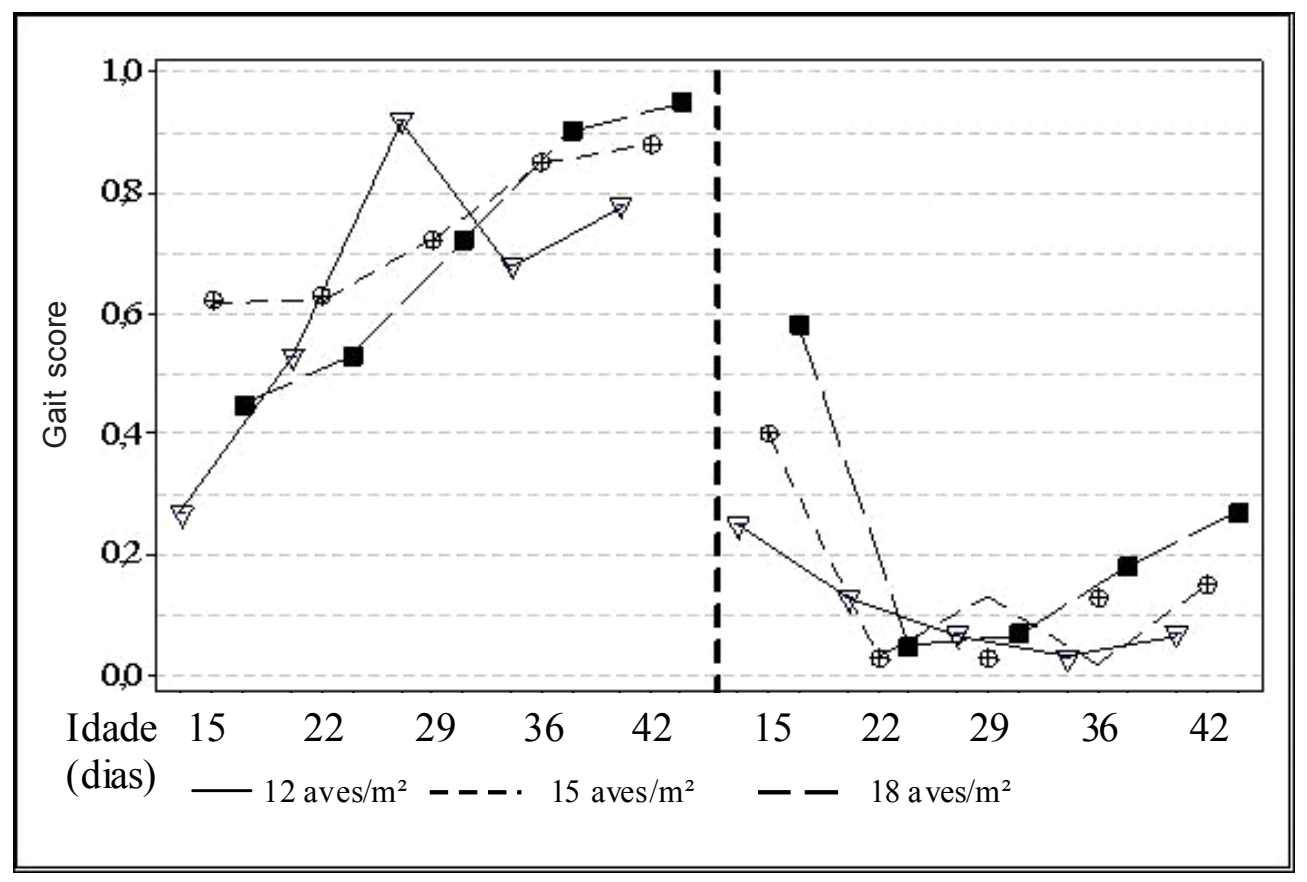

Figura 2. Escores médios obtidos para problemas locomotores para as metodologias (M1 e M2) para cada densidade e idade das aves. (Mean scores obtained for locomotor lesions for the methods (M1 and M2) for each density and age of the birds).

et al., 1997), podem afetar a habilidade de locomoção das aves. $\mathrm{O}$ fato mais importante para essas alterações é o aumento na intensidade de problemas ósseos e/ou diminuição progressiva da habilidade de locomoção com a idade. Conforme Kun et al. (2009) em estudos realizados com frangos de corte, a densidade de alojamento causou efeito significativo em algumas lesões da carcaça. Para os autores, ficou claro que a incidência de fraturas ósseas e articulares aumentou com o aumento da densidade de alojamento.

Weeks et al. (2000) relataram relação direta entre altos escores locomotores e redução da atividade das aves. Os autores registraram comportamentos de frangos de corte de 39 a 49 dias de idade, que obtiveram distintos escores e concluíram que as aves lesionadas (gait scores de 2 e 3 ) passaram mais tempo descansando e mais tempo em pé e se locomovendo, em comparação as aves com melhor habilidade locomotora (gait scores de 0 e 1) e, também reduziram o número de visitas ao comedouro. Aves lesionadas (gait score $>1$ ) realizaram em menor quantidade o comportamento de tomar banho de areia com o aumento da idade, em comparação as aves sadias (Vestergaard e Sanotra, 1999).

Em um estudo sobre o comportamento de frangos de corte, os autores observaram que as aves previamente estressadas apresentaram uma maior latência locomotora (intervalo de tempo até que a ave inicie a caminhar). Isso indica que situações estressantes de diversas naturezas afetam a habilidade locomotora das aves (Marin e Martijena, 1999).

Quanto à metodologia adotada, houve 
diferenças $(p<0,05)$ para quase todas as idades das aves avaliadas, exceto aos 15 dias de idade. Esse resultado pode ser explicado por essa ser a idade em que as aves apresentam incidência menor de problemas locomotores, indicando que, dependendo da metodologia adotada, as avaliações mostraram diagnósticos diferentes, ocasionando interpretações errôneas sobre o bemestar das aves.

Já para o efeito do avaliador, a diferença constatada nas avaliações foi observada somente para a idade de 36 dias $(\mathrm{p}<0,05)$ e para a metodologia 1 , demonstrando, que no geral, as metodologias são acessíveis de serem interpretadas e aplicadas.

Por meio da figura 2, observou-se que para metodologia 1 (habilidade locomotora), houve aumento gradativo na incidência de problemas locomotores com o desenvolvimento das aves. Quanto a metodologia 2 (resistência em permanecer em pé), verificou-se que as aves mais jovens apresentaram altos escores (baixa resistência em permanecer em pé, devido ao sistema locomotor estar ainda em desenvolvimento), se estabilizando na faixa de idade de 22 a 36 dias. Após essa idade, houve aumento da incidência de problemas locomotores, igualmente ao ocorrido com a metodologia 1.

Segundo Sorensen et al. (2000) têm-se discutido as estruturas e características essenciais para a aplicação de sistema adequado de previsão de bem-estar animal, sugerindo-se que os métodos de avaliação devem ser simples para mensurar; objetivos, frente a distintos avaliadores, produzir o mesmo resultado; sensíveis, que consigam refletir pequenas alterações no bem-estar animal; práticos e aplicáveis em condições reais de campo e, que realmente representem o bem-estar do animal.

A análise dos valores de ICC (índice de correlação intra-classe) para a confiabilidade interexaminadores mostrou, novamente, que os escores obtidos pelas distintas metodologias apresentaram níveis não aceitáveis de confiabilidade, para pelo menos um avaliador (tabela VI).

Logo, supõe-se que não há, atualmente, uma metodologia precisa para avaliação à campo do nível de bem-estar das aves, em função da incidência de problemas locomotores e de lesões no coxim plantar. O avanço na área da avicultura exige metodologias práticas, rápidas e que sirvam como padrão e linguagem única entre países auditados sobre as normas e legislação de produção de frangos de corte. Mulder e Hupkes (2007), relataram a importância de cumprimento das normas de produção de aves, bem como, as tendências futuras de atendimento ao bem-estar das aves e da qualidade do produto final, justificando a necessidade de estudos nessa área.

Tabela VI. Valores de ICC, duas vezes o erro padrão e nível de confiabilidade para os escores obtidos interexaminadores para as metodologias M1 e M2. A média representa os escores obtidos a partir dos três avaliadores. (ICC values, twice standard error and level of reliability for scores (intra-examiner) obtained using M1 and M2 methodologies. The average represents the scores obtained from the three evaluators).

\begin{tabular}{cccccc}
\hline Avaliador & Metodologia & Média & ICC & $2 \times$ erro-padrão & Nível \\
\hline 1 & 1 & 0,61 & 1,000 & 0,122 & Excelente \\
1 & 2 & 0,16 & 0,669 & 0,092 & Não aceitável \\
2 & 1 & 0,79 & 0,669 & 0,152 & Não aceitável \\
2 & 2 & 0,2 & 1,000 & 0,083 & Excelente \\
3 & 1 & 0,68 & 0,443 & 0,144 & Não aceitável \\
3 & 2 & 0,13 & 0,274 & 0,075 & Não aceitável \\
\hline
\end{tabular}

Archivos de zootecnia vol. 61, núm. 234, p. 226. 


\section{MENSURAÇÃO DE LESÕES LOCOMOTORAS EM FRANGOS DE CORTE}

\section{CONCLUSÕES}

Altas densidades de alojamento afetam a intensidade de problemas locomotores e de lesões no coxim plantar das aves, aumentando gradativamente com o desenvolvimento das mesmas.

As metodologias avaliadas para a mensuração de problemas locomotores e lesões no coxim plantar são passíveis de

\section{BIBLIOGRAFIA}

Berg, C. and Sanotra, G.S. 2003. Can a modified latency-to-lie test be used to validate gait-scoring results in commercial broiler flocks. Anim Welfare, 12: 655-659.

Bilgili, S.E., Montenegro, G.I., Hess, J.B. and Eckman, M.K. 1999. Sand as litter for rearing broiler chickens. J Appl Poultry Res, 8: 345351.

Cordeiro, A.F.S., Nääs, I.A. and Salgado, D.D. 2009. Field evaluation of broiler gait score using different sampling methods. Braz J Poultry Sci, 11: 140-154.

Cravener, T.L., Roush, W.B. and Mashaly, M.M. 1992. Broiler production under varying population densities. Poultry Sci, 71: 427-433.

Cummings, T.S., Stayer, P.A. and Webster, A.B. 2005. Field assessment of walking ability in commercial broilers. Poultry Sci, 84: S114.

Danbury, T.C., Weeks, C.A., Chambers, J.P., Waterman-Pearson, A.E. and Kestin, S.C. 2000. Self-selection of the analgesic drug carprofen by lame broiler chickens. Vet Rec, 146: 307311.

Dawkins, M.S., Donnelly, C.A. and Jones, T.A. 2004. Chicken welfare is influenced more by housing conditions than by stocking density. Nature, 427: 342-344.

Dozier, W.A., Thaxton, J.P., Branton, S.L., Morgan, G.W., Miles, D.M., Roush, W.B., Lott, B.D. and Vizzier-Thaxton, Y. 2005. Stocking density effects on growth performance and processing yields of heavy broilers. Poultry Sci, 84: 13321338.

Ekstrand, C. 1994. Kartliiggning av fothalsan hos svenska slaktkycklingar, och dess samband med olika miljijfaktorer. SLU/Hyg Specialarbete 23. Skara. $22 \mathrm{pp}$ serem aplicadas, porém não tão acessíveis de serem interpretadas e necessitam de detalhado treinamento para uso.

\section{AGRADECIMENTOS}

À Agroavícola Marostin de Dois Vizinhos/PR, pelo fornecimento dos animais e da ração, bem como com a confiança em nosso trabalho.

Ekstrand, C., Algers, B. and Svedberg, J. 1997. Rearing conditions and footpad dermatitis in Swedish broiler chickens. Prev Vet Med, 31: 167-174.

Estevez, I., Newberry, R.C. and De Reyna, L.A 1997. Broiler chickens: a tolerant social system? Etologia, 5: 19-29.

European Commission. 2000. The welfare of chickens kept for meat production (broilers). Report of the Scientific Committee on Animal Health and Animal Welfare. Brussels. 150 pp.

Garner, J.P., Falcone, C., Wakenell, P., Martin, M. and Mench, J.A. 2002. Field report scoring system and its use in assessing tibial dyschondroplasia in broilers. Braz Poultry Sci, 43: 355363.

Goliomytis, M., Panopoulou, E. and Rogdakis, E. 2003. Growth curves for body weight and major component parts, feed consumption, and mortality of male broiler chickens raised to maturity. Poultry Sci, 82: 1061-1068.

Greene, J.A., McRacken, R.M. and Evans, R.T. 1985. A contact dermatitis of broilers-clinical and pathological findings. Avian Pathol, 14: 2338.

Haslam, S.M. and Kestin, S.C. 2004. Comparing welfare in different systems. In: Weeks, C., Butterworth, A. (Orgs.). Measuring and auditing broiler welfare. CABI Publishing. USA. pp. 183195.

Havenstein, G.B., Ferket, P.R. and Qureshi, M.A. 2003. Growth, livability, and feed conversion of 1957 versus 2001 broilers when fed representative 1957 and 2001 broiler diets. Poultry Sci, 82: 1500-1508.

lunes, D.H., Castro, F.A., Salgado, H.S., Moura, I.C., Oliveira, A.S. e Bevilaqua-Grossi, D. 2005. 


\section{MENDES, PAIXÃO, MAROSTEGA, RESTELATTO, OLIVEIRAE POSSENTI}

Confiabilidade intra e terexaminadores e repetibilidade da avaliação postural pela fotogrametria. Rev Bras Fisiot, 9: 327-334.

Kestin, S.C., Adams, S.J.M. and Gregory, N.G. 1994. Leg weakness in broiler chickens, a review of studies using gait scoring. Proceedings of the $9^{\text {th }}$ European Poultry Conference. UK Branch of WPSA II. Glasgow. pp. 203-206.

Kestin, S.C., Knowles, T.G., Tinch, A.E. and Gregory, N.G. 1992. Prevalence of leg weakness in broiler chickens and its relationship with genotype. Vet Rec, 131: 190-194.

Kun, Z., Uluocak, A.N. and Karaman, M. 2009. The influence of some factors on carcass defects during fattening period in broilers. Arch Zootec, 58: 117-120.

Lewis, N.J. and Hurnik, J.F. 1990. Locomotion of broiler chickens in floor pens. Poultry Sci, 69 : 1087-1093.

Marín, R.H. e Martijena, I.D. 1999. Consecuencias de la exposición a un estresante sobre el comportamiento de pollos preseleccionados en un laberinto en T. Arch Zootec, 48: 405-414.

McGeown, D., Danbury, T.C., Waterman-Pearson, A.E. and Kestin, S.C. 1999. Effect of carprofen on lameness in broiler chickens. Vet Rec, 144: 668-671.

McWard, G.W. and Taylor, D.R. 2000. Acidified clay litter amendment. J Appl Poultry Res, 9: 518-529.

Mulder, R.W.A.W. and Hupkes, H. 2007. European legislation in relation to food safety in production of poultry meat and eggs. J Appl Poultry Res, 16: 92-98.
Neto, M.G. e Campos, E.J. 2002. Incidência de ascite em frangos de corte alimentados com rações comerciais de alto nível energético. Pesqui Agropecu Bras, 37: 1205-1212.

Roll, V.F.B., Dai Prá, M.A., Roll, A.A.P., Xavier, E.G., Rossi, P., Anciuti, M.A. e Rutz, F. 2010. Influência da altura de comedouros tubulares no comportamento ingestivo de frangos de corte. Arch Zootec, 59: 115-122.

Sorensen, P., SU, G. and Kestin, S.C. 2000. Effects of age and stocking density on leg weakness in broiler chickens. Poultry Sci, 79: 864-870.

SAS. 2003. Institute Inc. SAS OnlineDoc®. Version 8. SAS Institute Inc. Cary, NC. <http:// smoo.ciagri.usp.br/sasdoc/sasdoc/sashtml/ onldoc.htm> (10/06/2005).

Sugeta, S.M., Giachetto, P.F., Malheiros, E.B., Macari, M. e Furlan, R.L. 2002. Efeito na restrição alimentar quantitativa sobre o ganho compensatório e composição da carcaça de frango. Pesqui Agropecu Bras, 37: 903-908.

Vestergaard, K.S. and Sanotra, G.S. 1999 Relationships between leg disorders and changes in the behaviour of broiler chickens. Vet Rec, 144: 205-209.

Webster, A.B., Fairchild, B.D., Cummings, T.S. and Stayer, P.A. 2008. Validation of a three-point gait-scoring system for field assessment of walking ability of commercial broilers. J Appl Poultry Res, 17: 529-539.

Weeks, C.A., Danbury, T.D., Davies, H.C., Hunt, P. and Kestin, S.C. 2000. The behaviour of broiler chickens and its modification by lameness. Appl Anim Behav Sci, 67: 111-125.

Archivos de zootecnia vol. 61, núm. 234, p. 228. 